\title{
The Causes of Dizziness in Parkinson's Disease
}

\author{
Leyla Çavdar Yilmaz', Tugba Tunç², Levent E. İnan ${ }^{3}$ \\ ${ }^{1}$ Department of Neurology, Ministry of Health Trabzon of Hospital, Trabzon, Turkey \\ ${ }^{2}$ Department of Neurology, Faculty of Medicine, Gazi University, Ankara, Turkey \\ ${ }^{3}$ Department of Neurology, Ministry of Health Ankara Training and Research Hospital, Ankara, Turkey \\ Email: tugbatunc@yahoo.com
}

Received 25 March 2014; revised 24 April 2014; accepted 15 May 2014

Copyright (C) 2014 by authors and Scientific Research Publishing Inc.

This work is licensed under the Creative Commons Attribution International License (CC BY).

http://creativecommons.org/licenses/by/4.0/

cC) (7) Open Access

\begin{abstract}
Patients with Parkinson's disease complain dizziness frequently. Postural hypotension and postural instablity are suggested to be the reasons of dizziness in elderly people with Parkinson's disease. The aim of this prospective surveillance study was to characterize the etiology of the dizziness in patients with PD. A total of 47 consecutive patients with idiopathic Parkinson's disease were enrolled in the prospective surveillance study. Of the 47 patients, $32(68.1 \%)$ had dizziness. All patients underwent neurological and otologic examination. Complete blood count, serum electrolytes, glucose, liver function tests, renal function tests and thyroid function tests, cranial computerized tomography, direct cervical region radiography and vertebral artery doppler ultrasonography were obtained from all patients. Patients were screened for blood pressure changes, drug use, cardiovascular diseases, hypertension and diabetes mellitus. The findings of loss of disk height, with subsequent loss of cervical lordosis were higher in patients with dizziness $(p=0.01)$. The results of this study show that cervical-vertebral degeneration may be a common cause of dizziness in Parkinson's disease patients.
\end{abstract}

\section{Keywords}

Parkinson's Disease, Vertigo, Dizzness

\section{Introduction}

Parkinson's disease (PD) is associated with progressive neuronal loss of the substantia nigra and other brain structure. Although motor features such as tremor, rigidity and slowness of movements occur as main symptoms of disease, non-motor features including dementia, dysautonomia, and psychiatric complaining were established commonly [1]. Parkinson's disease is an age-related disorder and affects $1.5 \%$ of elderly population in 
Europe [2]. Dizziness is a common complaint which affects about $30 \%$ of people over the age of 65 years. Dizziness includes sensations such as faintness, light headedness, vertigo and imbalance [3]. For constituting the balance system, sensory inputs, integrating system or effector organs must be intact... Alterations in functions of one of these systems cause the dizziness or vertigo. Sensory inputs accepted from vestibular apparatus, visuel system, and proprioceptive system pass into the central nervous system and are integrated by the activity of the cerebellum, the extrapyramidal system, the limbic systwem and cerebral cortex [4]. Causes of dizzeness are neuro-otologic problems, general medical problems (cardiovascular disorders, drugs), cervical vertigo and multisensory dizziness syndrome [4]. The most common diagnosis reported in reffered patients is peripheral vestibular disease [3].

According to our observation, elderly patients with PD complain dizziness frequently. It is recognized that postural hypotension and postural instablity are the reasons of dizziness in elderly people with Parkinson's disease [5]. The aim of this prospective surveillance study was to characterize the etiology of the dizziness in patients with PD.

\section{Methods}

\subsection{Patients}

A total of 47 consecutive patients with idiopathic parkinson's disease who were examined at the movement disorders outpatient clinics in the Department of Neurology at Ankara Research Hospital from 2004 to 2006 were included in the study. A prospective study was designed, and the medical ethics committee of the hospital approved the study protocol. Informed consent was obtained from each participant according to the Declaration of Helsinki [6].

All patients were evaluated by the same neurologist who was blinded to presence of dizziness syptoms of patients. The diagnosis of PD was established according to the U.K. Parkinson's Disease Society Brain Bank criteria for idiopathic PD (UKP-DSBB) [7]. Patients with Parkinson-plus sendrome, secondary parkinsonism (drugs, vascular), patients with hearing loss, visual loss, acute or chronic otitis media were excluded. Patients with lesions such as infarction or space occupying lesions apparent on cranial computerized tomography were also excluded.

\subsection{Evaluation of Patients}

All patients were examined by the same neurologist that was blind to the history of dizziness of the patients and all exeminations were done while patients were "on" state. A total of 47 patients were underwent a neurological, eye movement, visual acuity, cerebellar system and, neuro-otological examination. The severity of disease was evaluated by using UPDRS and Hoehn and Yahr Scales [8]. In addition, each patient were assessed with the standart sheets include datas about the demographics, disease and dizziness symptoms. A list of medications taken by each patients was recorded. Additional diseases such as hypertension, diabetes mellitus, coronary artery diseases were also recorded. A dizzy patient was defined as one who described episodes of disequilibrium without fall.

For vestibulospinal system; Romberg test, Unterberger's tests, Tandem gait test, and gait test were applied by the neurogist [9]. For vestibulo-ocular function; nystagmus, gaze abnormalities, oculocephalic test, doll's eye manoeur, head impulse test, Hallpike test, past-pointing test [9] [10] were done. Complete otological examinations were made by the same otoneurologist.

Routine blood biochemistry tests, complete blood count and thyroid function tests and cranial computed tomography were performed for all patients.

Electrocardiographies of all patients were evaluated for cardiac arythmia. Blood pressure and heart rate were recorded using flat manometer instrument (ERKA perfect Aneroid Blue Line, Germany) Blood pressure was first measured after a 15 minute rest in a supine position. Patients were then asked to spontaneusly stand up and blood pressure was recorded after three minutes. A decrease $\geq 20 \mathrm{~mm} \mathrm{Hg}$ in systolic blood pressure, $\geq 10$ in diastolic blood pressure and, an increase $\geq 15$ beats in second in peripheral artery pulses were choosen as the criterion for orthostatic hypotension [11]. The differences in systolic, diastolic and peripheral arterial pulse between patients with and without dizziness were compared.

A total of 47 patients were investigated with color Doppler ultrasonography for vertebral artery insufficiency. 
Luminal diameter and mean velocity were calculated for each patient as existens. Vertebral artery evaluation was made as patient's neck was in anatomical posture. Same radiologists who were blind to the dizziness symptoms of patients evaluated the patients. Values of these parameters were compared in patients with and without dizziness.

All patients were underwent a direct radiographic evaluation of cervical region. Planes were taken anteriorposterior and lateral view. Loss of disk height, with subsequent loss of cervical lordosis, presence of anterior osteophytes and posterior osteophytes, reduced space between the vertebrae of neck were determined by the same radiologists [12]. Radiologist noted as "present” or "absent” for these abnormalities in direct radiographies of cervical region. In addition for the evaluation of vestibular nerve, odiometric tests were done.

\subsection{Statistical Analysis}

Statistical analysis was performed with SPSS for Windows, version 11.5 (SPSS, Chicago, IL). Variables were compared via the independent simple t test, statistical significance was defined as $\mathrm{p}<0.05$.

\section{Results}

A total of 47 patients met the inclusion criteria of the study. The overall mean age was $70.02 \pm 7.45$ years (range 51 to 82 years); 16 patients were female (34\%) and 31 were male (66\%). The overall mean duration of disease, according to the history obtained at diagnosis, was $49.58 \pm 48.16$ months (range 3 to 240 months).

Mean UPDRS scores of patients were $25.60 \pm 11.04$ (range 4 to 50). A total of 47 patients, 18 patients (39.1\%) were in Hoehn-Yahr phase 1, 25 patients (54.3\%) were in Hoehn-Yahr phase 2, 4 patients (8.51\%) were in Hoehn-Yahr phase 3. A total of 47 patients, 5 (10.9\%) had no medication, 4 (8.7\%) had only levodopa, 8 (17.4\%) had only dopamine agonists and 30 (63. 82\%) had combination of levodopa and dopamine agonist.

Of the 47 patients, 32 (68.1\%) had dizziness. The mean age of patients with dizziness was $71.78 \pm 4.88$ (range 60 - 82 years). The mean age of patients without dizziness was $66.26 \pm 10.83$ (range 51 - 82 years) (Table 1).

Between the patients with and without dizziness, there were significant differences between disease duration $(p=0.02)$ and mean UPDRS score $(p=0.009)$. Between the patients with and without dizziness, there were no significant differences in terms of age $(p=0.07)$, gender $(p=0.14)$, medications for Parkinson disease $(p=0.97)$, additional medications $(p=0.85)$, presence of hipertension $(p=0.53)$, diabetes mellitus $(p=0.7)$, coronary artery disease $(p=0.65)$ and dementia $(p=0.55)$ (Table 1$)$.

There were no significant differences in terms of cerebellar tests $(p=0.33)$, serum Na $(p=0.62), K(p=0.19)$, Cl ( $p=0.89)$, Ca $(p=0.92)$, free T3 (p = 0.93), free T4 (0.85), TSH $(p=0.78), H b(p=0.77), H c t(p=0.72)$, glucose $(p=0.23)$, urea $(p=0.14)$, creatine $(p=0.46)$, alanine ALT $(p=0.1)$, AST $(p=0.5)$, cholesterole $(p=$ $0.6)$, trigliseride $(p=0.2)$, cranial computed tomography $(p=0.95)$ between patients with and without dizziness (Table 2 and Table 3 ).

Between patients with and without dizziness, there was no significant differences in luminal diameters of righ and left vertebral arteries $(\mathrm{p}=0.41$ and 0.19 respectiveley) and mean blood flow velocities of right and left vertebral artery ( $\mathrm{p}=0.87$ and 0.07 respectively) (Table 4).

Table 1. Features of Parkinson's patients with and without dizziness.

\begin{tabular}{lcc}
\hline & Parkinson's disease with dizziness $(\mathrm{n}=32)$ & Parkinson's disease without dizziness $(\mathrm{n}=15)$ \\
\hline Age (years) & $71.78 \pm 4.88$ & $66.26 \pm 10.83$ \\
Sex (female/male) & $13 / 19$ & $3 / 12$ \\
Disease duration (month) & $58.51 \pm 53.34$ & $31.13 \pm 28.60$ \\
UPDRS & $28.51 \pm 10.61$ & $19.6 \pm 9.67$ \\
Hipertension & 16 & 6.02 \\
Diabetes mellitus & 3 & 0.009 \\
Additional drug therapy & 14 & 0.5 \\
Coronary artery disease & 6 & 0.7 \\
\hline
\end{tabular}


Table 2. Symptoms and signs of patients.

\begin{tabular}{|c|c|c|c|}
\hline $\begin{array}{c}\text { Symptoms and signs of } \\
\text { patients (n) }\end{array}$ & $\begin{array}{l}\text { Parkinson's disease with } \\
\text { dizziness }(\mathrm{n}=32)\end{array}$ & $\begin{array}{l}\text { Parkinson's disease without } \\
\text { dizziness }(\mathrm{n}=15)\end{array}$ & $\mathrm{p}$ \\
\hline Nausea & 7 & 4 & 0.8 \\
\hline Decrease in hearing & 15 & 7 & 0.09 \\
\hline Tinnitus & 13 & 2 & 0.03 \\
\hline Feeling of fullnes in the ear & 10 & 1 & 0.02 \\
\hline Romberg test abnormality & 0 & 0 & \\
\hline Tandem gait abnormality & 2 & 1 & 0.9 \\
\hline Unterberger's test abnormality & 1 & 0 & 0.5 \\
\hline Gaze abnormalities & 0 & 0 & \\
\hline Oculocephalic test abnormality & 0 & 0 & \\
\hline Head impulse test abnormality & 0 & 0 & \\
\hline Hallpike test abnormality & 0 & 0 & \\
\hline Presence of dysmetry & 2 & 0 & 0.1 \\
\hline Holmes's rebound test abnormality & 0 & 0 & \\
\hline Presence of dysdiadochokinesis & 0 & 0 & \\
\hline İntension tremor & 7 & 5 & 0.9 \\
\hline Presence of dysmetria & 2 & 0 & 0.1 \\
\hline Ataxia & 1 & 0 & 0.3 \\
\hline Nystagmus & 0 & 0 & \\
\hline
\end{tabular}

Table 3. The summary of the laboratuary tests results of patients.

\begin{tabular}{lccc}
\hline \multicolumn{1}{c}{ Laboratuary tests of blood } & Parkinson's disease with dizziness $(\mathrm{n}=32)$ & Parkinson's disease without dizziness $(\mathrm{n}=15)$ & $\mathrm{p}$ \\
\hline Hemoglobine (mg/dl) & $13.9 \pm 1.49$ & $40.89 \pm 3.83$ & 0.7 \\
Hematocrite (\%) & $40.42 \pm 4.29$ & $14.03 \pm 1.29$ & 0.7 \\
Glucose (mg/dl) & $110.25 \pm 26.17$ & $101.35 \pm 13.13$ & 0.2 \\
Urea (mg/dl) & $39.71 \pm 16.82$ & $33.04 \pm 5.91$ & 0.1 \\
Creatine (mg/dl) & $0.69 \pm 0.96$ & $0.9 \pm 0.22$ & 0.4 \\
Alanine transferase (mg/dl) & $17.06 \pm 5.9$ & $20.13 \pm 7.33$ & 0.1 \\
Aspartate transferase (mg/dl) & $19.09 \pm 6.27$ & $23.86 \pm 4.86$ & 0.1 \\
Sodium (g/mol) & $140.22 \pm 2.62$ & $140.62 \pm 2.51$ & 0.6 \\
Potassium (g/mol) & $4.42 \pm 0.5$ & $4.23 \pm 0.2$ & 0.1 \\
Calcium (g/mol) & $10.4 \pm 3.37$ & $10.4 \pm 3.77$ & 0.9 \\
Free triiodotyronine & $1.73 \pm 1.07$ & $1.72 \pm 0.89$ & 0.9 \\
Free thyroxine & $2.68 \pm 0.43$ & $2.66 \pm 0.52$ & 0.3 \\
Thyroid stimulating hormone & $1.43 \pm 1.28$ & $1.34 \pm 0.31$ & 0.3 \\
Cholesterole (mg/dl) & $194.82 \pm 35.6$ & $109.58 \pm 24.62$ & $0.83 \pm 44.16$ \\
Thrigliseride (mg/dl) & $122.55 \pm 47.32$ & & 0.2 \\
\hline
\end{tabular}


A decrease of at least $20 \mathrm{~mm} \mathrm{Hg}$ systolic blood pressure values was found in 1 (\%) of the 47 patients. A decrease of at least $10 \mathrm{~mm} \mathrm{Hg}$ diastolic blood pressure was found in 6 (\%) of the 47 patients. There were no significant decrease in systolic or diastolic blood pressure at standing in patients with dizziness $(\mathrm{p}=0.2$ and $\mathrm{p}=$ 0.2 respectively). On the contrary, significant increase in systolic and diastolic blood pressure were found (Table 5).

The findings of loss of disk height, with subsequent loss of cervical lordosis were higher in patients with dizziness $(\mathrm{p}=0.01)$. Of the 47 patients, $30(68.2 \%)$ had these abnormalities on the direct cervical radiography. Of the 30 patients with abnormality on the graphies, 24 (80\%) had dizziness.

The otologic examinations of patients were all normal but, 28 patients (59.2\%) were had mild sensoryneural hearing loss in odiologic examinations. But there were no differences in odiologic abnormalities between patients with and without dizziness $(\mathrm{p}=0.09)$.

\section{Discussion}

The results of this study show that cervical-vertebral degeneration may be a common cause of dizziness in Parkinson's disease patients. In the coordination of eye, head, and body posture, the proprioceptive inputs from neck are important [13]. Neck and head move together. So, disorder in cervicoproprioceptive mechanisms leads to unsteadiness or dizziness associated with neck movements. The head rotation sense is mediated by vestibular, proprioceptive or visual receptors. As well as, vestibular and visual disorders, neck proprioceptive abnormalities may induce dizziness. This proprioception is generated by the deep short intervertebral neck muscles that include extensive muscle spindles [14]. The unilateral irritation or deficit of neck afferents cause cervical tone imbalance and abnormalities of vestibular and neck inputs [13]. Thus degenerative disorders of cervical vertebrae may affect neck sensory input. In previous studies showed that the most common clinical causes of dizziness in older people in the community were central vascular disease or cervical spondylosis [15]. Degenerative changes of cervical uncovertebral junctions were assessed by anteroposterior direct radiographies of cervical region [16]. In fact magnetic resonance imaging (MRI) or computerized tomography (CT) of cervical spine are superior to direct radiographic images for disc, joint and bony abnormalities of cervical region. But this study was design as a surveillance investigations about the etiology of dizziness in PD. But Colledge et al. found no significant difference in the prevelance of the cervical cord and vertebrae in the subjects with and without dizziness [3].

Autonomic features are often together with PD. The involvement of the sympathetic system in the autonomic dysfunction of PD is well known antity. The occurence of orthostatic hypotension may be caused by the course of disease progression or dopaminergic drugs [17]. In one report the symptomatic orthostatic hypotension was

Table 4. The mean results of the color doppler ultrasonography of vertebral arteries.

\begin{tabular}{lcccccc}
\hline \multirow{2}{*}{ Vertebral arteries } & \multicolumn{2}{c}{ Parkinson's disease with dizziness $(\mathrm{n}=32)$} & \multicolumn{2}{c}{ Parkinson's disease without dizziness $(\mathrm{n}=15)$} & $\mathrm{p}$ & $\mathrm{p}$ \\
\cline { 2 - 6 } & Right & Left & Right & Left & Right & Left \\
\hline Luminal diameter $(\mathrm{mm})$ & $3.63 \pm 0.46$ & $5.65 \pm 4.20$ & $3.79 \pm 0.48$ & $3.77 \pm 0.42$ & 0.41 & 0.19 \\
Mean velocity $(\mathrm{ml} / \mathrm{sec})$ & $117.05 \pm 50.89$ & $145.22 \pm 50.1$ & $120 \pm 27.29$ & $112 \pm 18.22$ & 0.87 & 0.07 \\
\hline
\end{tabular}

Table 5. Summary of arterial blood pressure values of patients.

\begin{tabular}{lccc}
\hline & Lying & Standing & Change \\
\hline Mean systolic blood pressure (mmHg) & & & $\mathrm{p}$ \\
With dizziness & $144.6 \pm 25.3$ & $150.6 \pm 27.3$ & $5.93 \pm 3.45$ \\
Without dizziness & $125.3 \pm 9.9$ & $120.7 \pm 32.1$ & $2 \pm 0.46$ \\
Mean diastolic blood pressure (mmHg) & & & 0.22 \\
With dizziness & $87.6 \pm 10.3$ & $95.6 \pm 20.6$ & $5.93 \pm 3.6$ \\
Without dizziness & $76.6 \pm 4.8$ & $79.3 \pm 7.03$ & $2.6 \pm 1.03$ \\
\hline
\end{tabular}


found in duration of disease, daily levodopa and bromocriptine [18]. In PD, Lewy bodies was found in brain regions that include for autonomic nervous system such as locus coeruleus, dorsal vagal nucleus, intermediolateral nucleus [18]. In this study orthostatic hypotension was not found as the reason for dizziness of the patients with PD. But some reports did not support this result. Hiles et all. found that orthostatic hypotension was a major course of unexplained dizziness in elderly patients. However the occurence of orthostatic hypotension in PD remains controversial, some authors did not find higher incidence of orthostatic hypotension in course of PD [19] [20]. All patients underwent office measurements of lying and standing blood pressure. No patients were dizzy during the blood pressure measurements. Use of antihypertansive drugs and other additional medical problems were eliminated because groups were homogene for drugs and medical history.

Vertebrobasilar insufficiency (VBI) means poor circulation of blood in the vertebral and basillar arteries. There may be temporary block that leads to hypoperfusion of brain areas especially when the vertebral arteries kinked and narrowed by certain movements of the neck. This attacks may cause dizziness. In the case of hypotension, taking medication for hearth failure, PD can bring on a dizziness attack. Color doppler ultrasonography is the most widespread diagnostic procedure in obstructive disease of the arteries supplying the brain. In present study, there were not any differences in vertebral arteries doppler ultrasonographic parameters between patients with and without dizziness. Although in elderly the atherothrombotic events of extra and intracranial arteries are higher incidence, in our study group, vertebral insufficiency or flow disorders were not found as a reason of dizziness [21].

In recent reports, investigators showed that overall, 5.3\% of patients with PD have BPPV, and 10.8\% of patients who self-report dizziness have objective signs of BPPV. But in our research all patients undergo the vestibuler examinations were normal for vestibular tests [22]. Systemic disorders may cause to dizziness by affecting the sensory system, central system or target organs. Electrolyte disorders, hypothyroidism, anemia, hypoglicemia that may cause decrease cerebral perfusion, abnormalities of breathing, may result in dizziness. In this study these systemic causes was eliminated. There were some restrictive factors in this study. Magnetic resonance imaging of cervical region is superior to direct radiographic graphies. The most identical datas may obtain from electronystagmography of patients with dizziness for detecting the vestibular abnormalities. But this study was design as a surveillance study. According to the results of this study cervical degenerative changes were seem to the cause of the dizziness of patients with PD. Elderly people may commonly suffer from the symptoms of the cervical vertebral degenerations. As a suggestion, finding of high incidence of cervical vertebral degeneration as a cause of dizziness in patients with parkinson's disease may connected to the age of the patients. But on the other hand, if longer mean disease duration and higher mean UPDRS scores in patient with dizziness are to take in the consideration, dizziness may be accepted as one of the systemic symptoms of late Parkinson's disease. We suggested that for accepting the dizziness as a symptoms of the disease, there must be designed wide prospective and controlled studies.

\section{References}

[1] Gelby, D.J., Oliver, E. and Gilman, S. (1999) Diagnostic Criteria for Parkinson Disease. Archives of Neurology, 56, 33-39. http://dx.doi.org/10.1001/archneur.56.1.33

[2] de Rijk, M.C., Trourio, C., Breteler, M.M., Dortiques, J.F., Amaducci, L., Lopez-Pouse, S., Manubens-Bertran, J.M., Alperovitch, A. and Rocca, W.A. (1997) Prevalence of Parkinsonism and Parkinson's Disease in Europe: The Europarkınson Collaborative Study. European Community Concerted Action on the Epidemiology of Parkinson's Disease. Journal of Neurology, Neurosurgery \& Psychiatry, 62, 10-15. http://dx.doi.org/10.1136/jnnp.62.1.10

[3] Colledge, N., Lewis, S., Mead, G., Sellar, R., Wardlaw, J. and Wilson, J. (2002) Magnetic Resonance Brain İmaging in People with Dizziness: A Comparison with Nondizzy People. Journal of Neurology, Neurosurgery \& Psychiatry, 72, 587-589. http://dx.doi.org/10.1136/jnnp.72.5.587

[4] Luxon, L.M. (2004) Evaluation and Management of the Dizzy Patient. Journal of Neurology, Neurosurgery \& Psychiatry, 75, 45-52. http://dx.doi.org/10.1136/jnnp.2004.055285

[5] Barone, P., Antonini, A., Colosimo, C., et al. (2009) The PRIAMO Study: A Multicenter Assessment of Nonmotor Symptoms and Their İmpact on Quality of Life in Parkinson's Disease. Movement Disorders, 24, 1641-1649. http://dx.doi.org/10.1002/mds.22643

[6] International Committee of Medical Journal Editors (1991) Statements from the Vancouver Group. British Medical Journal, 302, 1194.

[7] Gibb, W.R. and Lees, A.J. (1988) The Relevance of the Lewy Body to the Pathogenesis of İdiopathic Parkinson's 
Disease. Journal of Neurology, Neurosurgery \& Psychiatry, 51, 745-752. http://dx.doi.org/10.1136/jnnp.51.6.745

[8] Siderowf, A., McDermott, M., Kieburtz, K., Blindauer, K., Plumb, S., Shoulsa, I., Parkinson Study Group (2002) Test-Retest Reliability of the Unified Parkinson's Disease Rating Scale in Patients with Early Parkinson's Disease: Results from a Multicenter Clinical Trial. Movement Disorders, 17, 758-763. http://dx.doi.org/10.1002/mds.10011

[9] Davies, R. (2004) Bedside Neuro-Otological Examination and İnterpretation of Commonly Used İnvestigations. Journal of Neurology, Neurosurgery \& Psychiatry, 75, 32-44. http://dx.doi.org/10.1136/jnnp.2004.054478

[10] Bronstein, A.M. (2003) Vestibular Reflexes and Positional Manoeuvres. Journal of Neurology, Neurosurgery \& Psychiatry, 74, 289-293. http://dx.doi.org/10.1136/jnnp.74.3.289

[11] Low, P.A. and Engstrom, J.W. (2005) Disorders of the Autonomic Nervous System. In: Kasper, D.L., Braunwald, E., Fauci, A.S., Hauser, S.L., Longo, D.L. and Jameson, J.L., Eds., Harrison's Principles of Internal Medicine, 16th Edition, Mc-Graw-Hill, New York, 2428-2434.

[12] Kelsey, J.L., Githens, P.B., Walter, S.D., Southwick, W.O., Weil, U., Holford, T.R., Ostfeld, A.M., Calogere, J.A., O’Connor, T. and White, A.A. (1984) An Epidemiological Study of Acute Prolapsed Cervical İntervertebral Disc. The Journal of Bone and Joint Surgery (American Volume), 66, 907-914.

[13] Brandt, T. and Bronstein, A.M. (2001) Cervical Vertigo. Journal of Neurology, Neurosurgery \& Psychiatry, 71, 8-12. http://dx.doi.org/10.1136/jnnp.71.1.8

[14] Richmond, F.J.R. and Bakker, D.A. (1982) Anatomical Organization and Sensory Receptor Content of Soft Tissues Surrounding Upper Cervical Vertebrae in the Cat. Journal of Neurophysiology, 48, 49-61.

[15] Colledge, N.R., Barr-Hamilton, R.M., Lewis, S.J., Sellar, R.J. and Wilson, J.A. (1996) Evaluation of İnvestigations to Diagnose the Course of Dizziness in Elderly People: A Community Based Controlled Study. British Medical Journal, 313, 788-792. http://dx.doi.org/10.1136/bmj.313.7060.788

[16] Cushnaghan, J. and Dieppe, P. (1991) Study of 500 Patients with Limb Joint Osteoarthritis. Analysis by Age Symptomatic Joint Sites. Annals of the Rheumatic Diseases, 50, 8-13. http://dx.doi.org/10.1136/ard.50.1.8

[17] Hilen, M.E., Wagner, M.L., Pharm, D. and Sage, J.I. (1996) Subclinical Orthostatic Hypotension Is Associated with Dizziness in Elderly Patients with Parkinson Disease. Archives of Physical Medicine and Rehabilitation, 77, 710-712. http://dx.doi.org/10.1136/ard.50.1.8

[18] Senard, J.M., Rai, S., Lapeyre-Mestre, M., Brefel, C., Rascol, O. and Montastruc, J.L. (1997) Prevalence of Orthostatic Hypotension in Parkinson's Disease. Journal of Neurology, Neurosurgery \& Psychiatry, 63, 584-589. http://dx.doi.org/10.1136/jnnp.63.5.584

[19] Sandroni, P., Ahlskog, J.E., Fealey, R.D. and Low, P.A. (1991) Autonomic İnvolvement in Extrapyramidal and Cerebellar Disorders. Clinical Autonomic Research, 1, 147-155. http://dx.doi.org/10.1007/BF01826212

[20] Martinez-Martin, P., Schapira, A.H.V., Stocchi, F., et al. (2007) Prevalence of Nonmotor Symptoms in Parkinson’s Disease in an İnternational Setting; Study Using Nonmotor Symptoms Questionnaire in 545 Patients. Movement Disorders, 22, 1623-1629. http://dx.doi.org/10.1002/mds.21586

[21] Leonetti, J.P. and Srinivas, M. (1997) Advances in the Diagnosis and Management of Vertebrobasilar İnsufficiency. Otolaryngology \& Head and Neck Surgery, 5, 272-275.

[22] van Wensen, E., van Leeuwen, R.B., van der Zaag-Loonen, H.J., Masius-Olthof, S. and Bloem, B.R. (2013) Benign Paroxysmal Positional Vertigo in Parkinson’s Disease. Parkinsonism \& Related Disorders, 19, 1110-1112. 
Scientific Research Publishing (SCIRP) is one of the largest Open Access journal publishers. It is currently publishing more than 200 open access, online, peer-reviewed journals covering a wide range of academic disciplines. SCIRP serves the worldwide academic communities and contributes to the progress and application of science with its publication.

Other selected journals from SCIRP are listed as below. Submit your manuscript to us via either submit@scirp.org or Online Submission Portal.
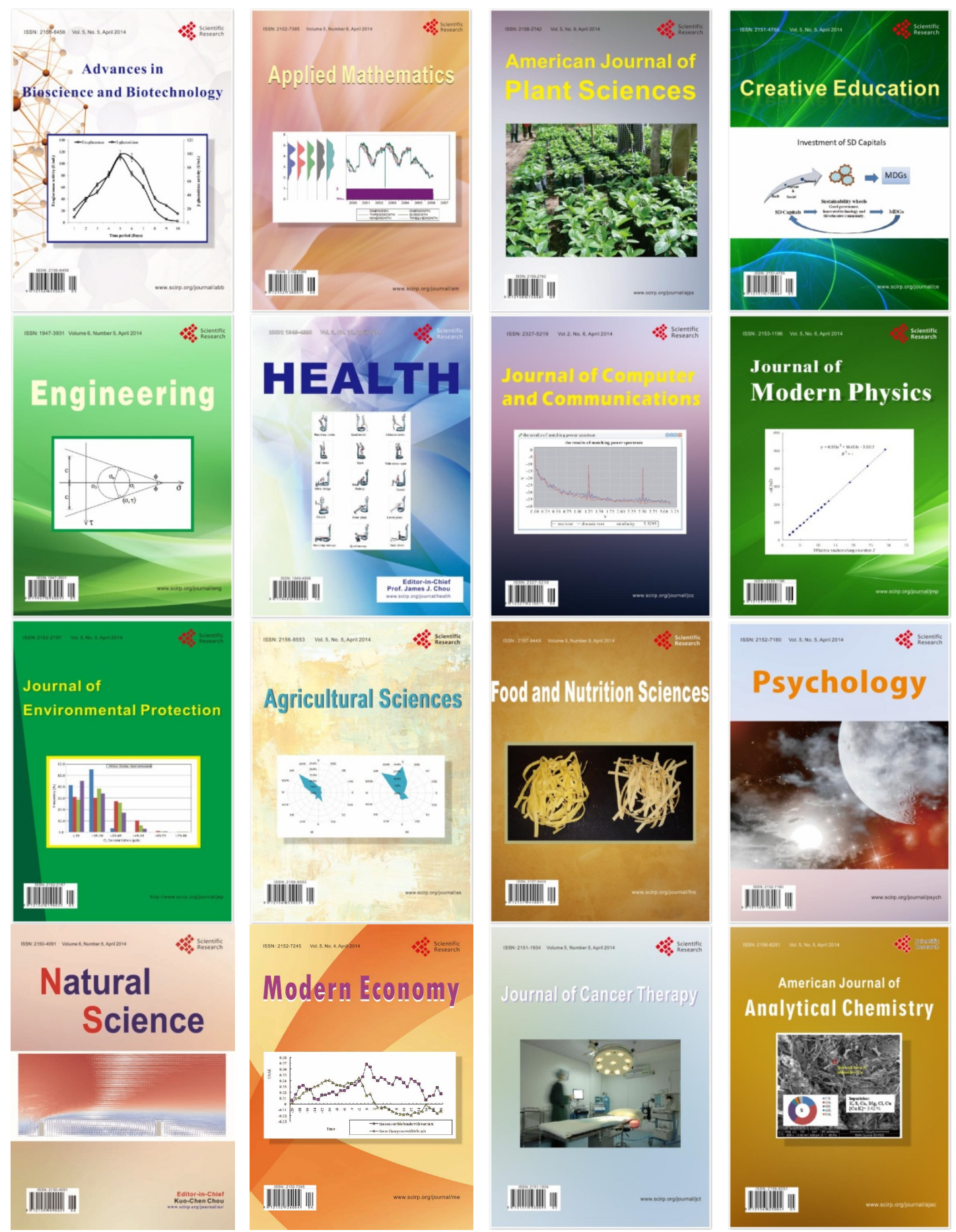